\title{
A Multimedia Terminal Supporting Adaptation for QoS Control
}

\author{
Beilu Shao ${ }^{1}$, Daniele Renzi ${ }^{2}$, Marco Mattavelli ${ }^{1}$, Stefano Battista ${ }^{2}$, Samuel Keller ${ }^{1}$ \\ ${ }^{1}$ Ecole Polytechnique Fédérale de Lausanne (EPFL), Switzerland \\ ${ }^{2} b$ Soft Ltd, Italy \\ beilu.shao@epfl.ch,daniele@bsoft.info,marco.mattavelli@epfl.ch, \\ bautz@bsoft.info,samuel.keller@epfl.ch
}

\begin{abstract}
This paper addresses the End-to-End Quality of Service (QoS) guarantees and control for multimedia content delivery over heterogeneous networks, with a particular focus on end user terminal perspective. A multimedia terminal which embeds different probes to monitor network conditions and end user perceptual characteristics has been conceived and implemented. A series of QoS mapping between perception and adaptation spaces are yielded by means of the parameters provided by the QoS probes, facilitating content adaptation enabled by any generic adaptation server or intermediate service. Such a design approach illustrates a possible architecture for next-generation multimedia end user system supporting QoS control and content adaptation over a heterogeneous delivery chain.
\end{abstract}

\section{Introduction}

The recent advances in multimedia computing and great success of the Internet have drawn tremendous attention and contribution from both academia and industry. Many different approaches have been developed to enable the efficient and effective multimedia content delivery, ranging from advanced video coding with outstanding rate-distortion performances up to reliable and adaptive video transmission. In the meanwhile, various kinds of communication and terminal devices have been deployed, such as PC, Set-Top Box and mobile devices like PDA. The multimedia content delivery has been growing from single format video transmission over internet to complex multimedia adaptive delivery across heterogeneous networks, terminals and users [1].

In particular, the Internet is growing from a monolithic data service network to the next-generation network convergence of services. An example is the deployment of real-time audiovisual services with
End-to-End QoS support through resources provisioning, monitoring and control. The End-to-End (E2E) QoS provisioning together with dynamic multimedia content adaptation, constitute a very promising approach to real-time multimedia applications [2].

Along this trend, new standards have emerged. MPEG-21 [3, 4] has been developed to provide a set of tools to enable "transparent and augmented use of multimedia resources across a wide range of networks and devices used by different communities". Of particular importance towards the fulfillment of this objective is the aspect of digital content adaptation. Part 7 of MPEG-21, Digital Item Adaptation, has been delivered specifically addressing this aspect $[5,6]$.

However, current multimedia terminal designs do not consider QoS requirements, thus they do not offer the necessary functionality. This paper investigates the multimedia end user terminal design to fulfill the Endto-End QoS control objectives for adaptive content delivery. A series of QoS mapping from perceptual QoS to adaptation QoS are developed. The latter approach is used to trigger an adaptation action whereby an Adaptation Decision-Taking Engine, for example, can select new service parameters to reduce the bit rate in case of limited network bandwidth capabilities.

The paper is organized as follows. Section 2 presents the various QoS levels involved in an end-toend QoS-based framework. The approaches of QoS mapping for adaptation feedback are also highlighted. Section 3 presents the integration of QoS probes into a Media Player. Section 4 presents the multimedia terminal middleware architecture and the interactions between the Media Player and QoS Probes. Section 5 briefly draws the conclusion.

\section{Subjective, Objective and Adaptation QoS}


So as to analyze the media content at the terminal side and to provide sufficient information for the adaptation modules, perceived Quality of Service (pQoS) probes have been developed at the user terminal. The measured values of perceived quality are periodically provided to the adaptation modules. Whenever quality degradation is detected, the terminal will generate an alert for adaptation modules to initiate a content adaptation decision process.



\section{Fig. 1. Subjective vs objective vs adaptation vs network QoS.}

During the process, a series of QoS mapping are calculated. Initially, an objective approximation of the perceptual quality, i.e. the quality as it is perceived by the user, that is the subjective QoS, is taken, in order to automate the overall process. Such an approximation is provided by the objective QoS, which is extracted from the objective quality parameters (e.g. bit rate, frame rate, packet loss, audio loudness, video colorfulness, etc.), at both terminal (objective pQoS) and network (network QoS) level. The objective QoS is mapped to subjective QoS by means of specific mapping algorithms, which have been extensively addressed and reported. Then, the objective QoS has to be mapped to the adaptation QoS (aQoS), according to agreed Service Level Agreements (SLA). Such a mapping makes a correspondence between quality levels measured at the terminal (objective QoS) and practical adaptation parameters (adaptation QoS), e.g. the bit rate, i.e. so as to make the adaptation modules aware of the application class (also named service level) that should correspond to each quality level measured at the terminal.

For adaptation, the quality information is provided in terms of absolute values of objective QoS, rather than in terms of alarms to leave the entire decision process to the adaptation modules. Therefore, the latter must be aware of the pQoS-aQoS mapping for each terminal. E.g. the same pQoS could correspond to a certain service level (e.g. Silver in section 2.2) for IPTV services, but higher levels (e.g. Gold) for UMTS services.

\subsection{Subjective QoS to Objective QoS Mapping}

A subjective evaluation of the quality of the content practically is a difficult task due to various factors including time, cost and human perception. Strictly speaking, subjective tests require a large number of tests operated under controlled psychometric experimental conditions, to obtain statistically meaningful Mean Opinion Scores (MOS), summarizing the pQoS. As alternatives, various objective quality assessment algorithms have been proposed.

Table 1. Subjective MOS and R Factor scale

\begin{tabular}{|l|c|c|}
\hline Subjective Interpretation & MOS & R Factor \\
\hline Excellent - almost all satisfied & 5 & 90 \\
\hline Very good - most users satisfied & 4 & 80 \\
\hline Good - some users unsatisfied & 3 & 70 \\
\hline Fair - many users unsatisfied & 2 & 60 \\
\hline Poor - most users unsatisfied & 1 & 50 \\
\hline
\end{tabular}

Objective QoS can be assessed by analyzing the signals in both compressed (e.g. MPEG-4 compressed video stream) and non-compressed (e.g. reconstructed RGB video to be output) formats, as shown in the Fig.1. A MOS rating ranged under a standard scale is usually used for subjective tests in [7] and summarized in the Table 1 . The objective MOS is based on the same scale, as stated in ITU-T P.800.1 [8]. As shown in Table 1, such a scale can be also mapped to $\mathrm{R}$ Factor ranges on a scale of $0-100$. The $\mathrm{R}$ Factor is the rating factor used by the E-model [9], which is another rating system especially used for conversational systems. E-model provides an objective measurement of quality, normally based on packet loss, jitter and delay as main quality parameters. Usually the R Factor is computed first from the objective parameters, using the following formula [10]:

$$
R=R_{o}-I_{s}-I_{d}-I_{e}+A
$$

where $R_{o}$ represents the basic signal-to-noise ratio; $I_{s}$ is the combination of all impairments occurring simultaneously with the signal, such as the quantization noise; $I_{d}$ represents the impairments caused by delay; $I_{e}$ is the equipment impairment factor, representing impairments caused by low bit rate codecs; $I_{e}$ also includes impairments due to randomness of packet loss; $A$ is the advantage factor. Based on the above formula, the correlation between $\mathrm{R}$ values, transmission quality category (MOS) and user satisfaction level (subjective interpretation) is defined. Therefore, the objective MOS and the $\mathrm{R}$ Factor provide a practical mapping between the measured quality parameters and the subjective quality. Such a mapping can be sustained by experimental results 
obtained from formal subjective tests performed using standard methods, such as the one presented in [11].

In many other cases, as the one in this multimedia terminal, proprietary formulas are used to directly map the objective parameters, including the media quality parameters, to the MOS, depending on the implementation and on the specific use cases. Indeed, the presence of multiple media and protocols can lead to the definition of different sets of QoS parameters, specifically suitable for each context. Moreover, several types of probes are available in the market, each one relying on different probing methods. That can make a probe useful for a specific context and useless for another one. The level of correlation between the objective QoS and the subjective QoS provided by a probe by means of such formulas represents the main added value of all commercial pQoS probes.

\subsection{Perceived QoS to Adaptation QoS Mapping}

The modules performing the adaptation decisionmaking process need to be provided with an adaptation QoS to perceived QoS mapping, to get a correspondence between the perceived QoS and the initial deployed service QoS (aQoS). Adaptation QoS relates to the facilities embedded within an application that preserve the quality of its intended usage, by providing the levels of application class (e.g. in terms of bit rate, frame rate, etc.), which correspond to the quality levels perceived by the user; e.g. each frame rate (aQoS) corresponds to a perceived quality (pQoS) level. Thus, they can make decisions on when to commute from a quality level to another one. A performance threshold can be set against each application class. Such a mapping can be agreed between the service provider and the probe constructor.

Usually three levels of aQoS are used (denoted here as Bronze, Silver, Gold), and any of them is mapped to a specific range of subjective or objective QoS (that we will generally address as pQoS) scores, measured in MOS. For example, in a simple scenario performing frame rate adaptation by temporal scalability, the frame rate is the main perceived QoS parameter; the perceived frame rate must correspond to an SVC temporal layer in the source stream: that is why a mapping, based on experimental results, between pQoS (perceived frame rate) and aQoS (SVC temporal layer) is needed, as example in Table 2.

Table 2. aQoS - pQoS mapping example

\begin{tabular}{|c|c|c|c|}
\hline aQoS & Gold & Silver & Bronze \\
\hline SVC Temporal Layer & 2 & 1 & 0 \\
\hline Frame Rate[fps] & 25.000 & 12.500 & 6.250 \\
\hline
\end{tabular}

\begin{tabular}{|c|c|c|c|}
\hline $\begin{array}{c}\text { Target pQoS } \\
\text { [R factor, scale 0 - 100] }\end{array}$ & 85 & 70 & 55 \\
\hline $\begin{array}{c}\text { pQoS Interval } \\
\text { [R factor, scale 0 - 100] }\end{array}$ & 10 & 10 & 10 \\
\hline $\begin{array}{c}\text { pQoS Range } \\
\text { [R factor, scale 0 - 100] }\end{array}$ & $80-90$ & $65-75$ & $50-60$ \\
\hline MOS [scale 1 - 5] & 4.4 & 3.8 & 3.2 \\
\hline
\end{tabular}

\subsection{Implementation of the Concepts}

2.3.1 Network and media QoS parameters. In the practical implementation of the concepts described in this paper, the QoS probes integrated in the Media Player perform a real-time "sniffing" of the incoming video packets, filter all the RTSP/RTCP/RTP streaming sessions, recognize the out-of-band setup information contained in the SDP, and then perform batch analysis, calculation and logging of the estimated quality parameters from relevant streaming packets. The objective QoS parameters monitored can be grouped into two domains: 1) Network quality domain parameters, i.e. objective network quality parameters, such as packet loss, jitter, delay, etc. 2) Media (Audio and Video) quality domain parameters, i.e. objective media quality parameters, such as bit rate, audio loudness, audio saturation, video frame size, video colorfulness, etc.

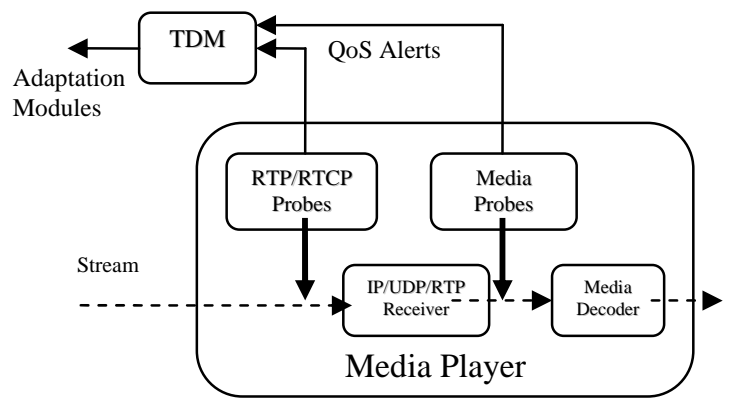

Fig. 2. QoS probes integrated in media player

2.3.2 QoS metrics generation. The QoS probes provide the Terminal Device Middleware (TDM) with the calculated Audio MOS and Video MOS values. Such information is then processed by the adaptation modules to generate alarms when the quality is degrading, then using the pQoS-aQoS mapping to make the adaptation decisions. The adaptation is finally performed by adjusting the application parameters in order to get the best possible quality, even according to the statistics provided by the network probes (network QoS, as shown in Fig.2.), which could be eventually present in the network framework. 


\section{Probes Integrated with Media Player}

The QoS probes are integrated with the Media Player, as shown in Fig. 3. The network probes detect quality information from the TCP/RTSP and UDP/RTP sessions, while the media probes interact with the audio and video decoder to extract media quality information, as described in section 2.

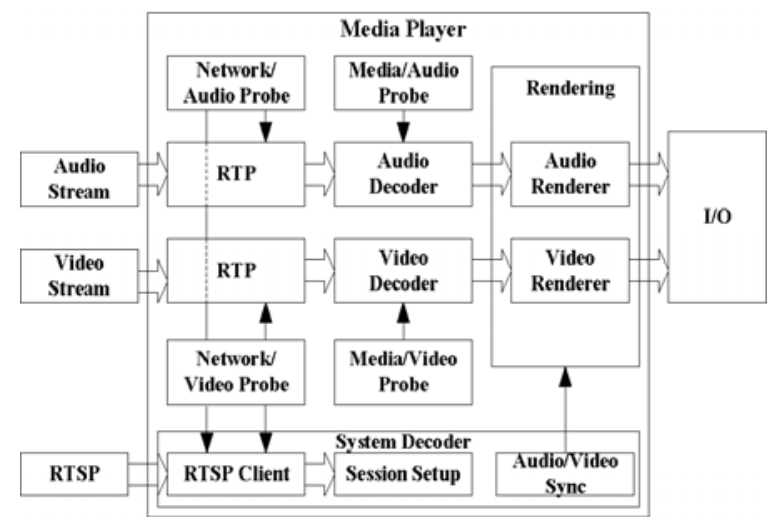

Fig. 3. Media player architecture

\section{Interaction with Multimedia Terminal}

The Media Player needs to be coordinated under a terminal middleware together with other modules to achieve fruitful user experiences. These modules include, but do not limit to a Digital Item Browser to browse and process content related information (e.g. MPEG-21 DIDs), a UED-UCD module to describe the user environments and user preferences in standard MPEG-21 form, and key management systems for intellectual property management and protection.

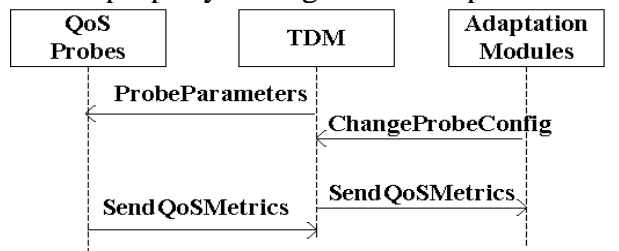

\section{Fig. 4. Interaction of TDM and media player}

The Terminal Device Middleware has two main tasks: 1) externally interacting with server or intermediate service, e.g. to feedback the QoS control for content adaptation, and 2) locally coordinating the convergence of Digital Item Browser, Media Player, UED-UCD modules, QoS Probes. In this paper, we focus only on the End-to-End QoS control problem and thus discuss only the terminal middleware support for the Media Player. The QoS parameters are exchanged through the TDM between QoS Probes and adaptation modules, as shown in Fig. 4. Such an exchange is performed periodically (e.g. every 10 seconds), and special alarms are sent back to the adaptation modules out of period, when certain conditions, such as the total absence of the signal, are detected in the terminal.

\section{Conclusion}

This paper presents a multimedia terminal architecture supporting content adaptation with Endto-End QoS control for adaptive multimedia delivery over heterogeneous networks and devices. This terminal includes several QoS probes which monitor the transmission and perceptual characteristics. A series of QoS mapping are specified so as to provide adaptation QoS value for adaptation modules. The terminal architecture integrates the terminal middleware, the media player and the related QoS probes.

\section{References}

[1] A. Vetro, C. Christopoulos, and T. Ebrahimi, eds. "Special Issue on Universal Multimedia Access", IEEE Signal Processing Magazine, vol.20 (2), Mar. 2003.

[2] T. Ahmed, A. Asgari, A. Mehaoua, E. Borcoci, L. B. Equille, and K. Georgios, "End-to-end quality of service provisioning through an integrated management system for multimedia content delivery", Computer Communications, vol.30 (2), 2007, 638-651.

[3] ISO/IEC 21000, "Information Technology - Multimedia Framework (MPEG-21)”, 2002.

[4] F. Pereira, J. R. Smith, and A. Vetro, eds., "Special Issue on MPEG-21”, IEEE Trans. on Multimedia, vol.7 (3), Jun. 2005.

[5] ISO/IEC TR 21000-7:2004 "Information Technology Multimedia Framework (MPEG-21)-Part7: Digital Item Adaptation", 2004.

[6] A. Vetro and C. Timmerer, "Digital Item Adaptation: Overview of Standardization and Research Activities", IEEE Trans. on Multimedia, vol.7 (3), Jun. 2005, 418-426.

[7] T. Alpert, V. Baroncini, D. Choi, L. Contin, R. Koenen, F. Pereira, and H. Peterson, "Subjective Evaluation of MPEG-4 Video Codec Proposal: Methodological Approach and Test Procedures”, Signal Processing: Image Communication, vol. 9, 1997, 305-325.

[8] ITU-T P.800.1, "Mean Opinion Score (MOS) terminology”, 2006.

[9] ITU-T G.107, "The E-model, a computational model for use in transmission planning”, 1998.

[10] ITU-T G.108, "Application of the E-Model - A planning guide”, 1999.

[11] T. Yamazaki, "Subjective video quality assessment for adaptive quality-of-service control", in Proc. IEEE ICME, Tokyo, Japan, Aug. 2001. 